\title{
Flat surfaces in hyperbolic 3-space whose hyperbolic Gauss maps are bounded
}

\author{
Francisco Martín, Masaaki Umehara and Kotaro Yamada
}

\begin{abstract}
We construct a weakly complete flat surface in hyperbolic 3 -space $H^{3}$ having a pair of hyperbolic Gauss maps both of whose images are contained in an arbitrarily given open disk in the ideal boundary of $H^{3}$. This construction is accomplished as an application of minimal surface theory. This is an interesting phenomenon when one compares it with the fact that there are no complete non-flat minimal (resp. non-horospherical constant mean curvature one) surfaces in $\mathbb{R}^{3}$ (resp. $H^{3}$ ) having bounded Gauss maps (resp. bounded hyperbolic Gauss maps).
\end{abstract}

\section{Introduction}

It is a classical fact that any complete immersed flat surface in the hyperbolic 3-space $H^{3}$ must be a horosphere or a hyperbolic cylinder, where 'flat' means that the Gaussian curvature vanishes identically. However, this does not imply the lack of an interesting global theory for flat surfaces. Gálvez, Martínez and Milán [7] established a Weierstrass-type representation formula for such surfaces. In this paper, we consider flat surfaces with admissible singularities (a singular -i.e., degenerate - point is called admissible if the corresponding points on nearby parallel surfaces are regularly immersed; see [10]). Flat surfaces with admissible singularities in $H^{3}=\mathrm{SL}(2, \mathbb{C}) / \mathrm{SU}(2)$ are called flat fronts, and can be characterized as the projections of holomorphic immersed Legendrian curves in $\operatorname{SL}(2, \mathbb{C})$. Here, a holomorphic map $\mathcal{L}: \mathbb{D}_{1} \rightarrow \mathrm{SL}(2, \mathbb{C})$ is called Legendrian if the pull-back $\mathcal{L}^{*} \Omega_{\mathrm{SL}}$ vanishes on $\mathbb{D}_{1}:=\{z \in \mathbb{C}:|z|<1\}$, where $\Omega_{\mathrm{SL}}$ is the complex contact form on $\mathrm{SL}(2, \mathbb{C})$ defined as

$$
\Omega_{\mathrm{SL}}:=x_{11} d x_{22}-x_{21} d x_{12} \quad\left(\left(x_{i j}\right)_{i, j=1,2} \in \mathrm{SL}(2, \mathbb{C})\right) .
$$

A flat front in $H^{3}$ induces a pair of hyperbolic Gauss maps $\left(G_{+}, G_{-}\right)$, both of which are holomorphic mappings into $\mathbb{C} \cup\{\infty\}$ as follows. Let $f: M^{2} \rightarrow H^{3}$ be a flat front

Mathematics Subject Classification (2010): Primary 53D10; Secondary 53A10, 53A35.

Keywords: Contact manifolds, minimal surfaces, flat fronts, improper affine fronts. 
defined on a Riemann surface $M^{2}$. The normal geodesic passing through $f(p)$ meets the ideal boundary $\partial H^{3}$ of the hyperbolic 3 -space $H^{3}$ at $G_{ \pm}(p)$ if one identifies $\partial H^{3}$ with $\mathbb{C} \cup\{\infty\}$ via the Poincaré half-space model of the hyperbolic 3 -space. In [10], the two hyperbolic Gauss maps $G_{+}$and $G_{-}$are denoted by $G$ and $G_{*}$, respectively. We are interested in the behavior of the hyperbolic Gauss maps of flat surfaces in $H^{3}$. For flat fronts in $H^{3}$, completeness and weak completeness are defined (cf. $\S 2$ of [12]). Completeness implies weak completeness. There are many complete or weakly complete flat fronts in $H^{3}$, as shown in [10], [11], and [12].

It is well known that the Gauss map (resp. hyperbolic Gauss map) of a complete immersed non-flat minimal surface in $\mathbb{R}^{3}$ (resp. a complete immersed nonhorospherical constant mean curvature one surface in $H^{3}$ ) can omit at most four points (see Fujimoto [5] and [6], and Yu [17]). It is well known that conformal minimal immersions are obtained by taking the real parts of null holomorphic immersions. Recently, as an improvement of Nadirashvili's discovery [14] of complete bounded minimal surfaces in $\mathbb{R}^{3}$, the existence of a complete bounded null holomorphic immersion

$$
F: \mathbb{D}_{1} \longrightarrow \mathbb{C}^{3}
$$

of the unit disk $\mathbb{D}_{1} \subset \mathbb{C}$ has been shown (see [1], and also [4]), where null means that $F_{z} \cdot F_{z}$ vanishes identically; here $F_{z}:=d F / d z$ is the derivative of $F$ with respect to the complex coordinate $z$ of $\mathbb{D}_{1}$ and the dot denotes the canonical complex bilinear form. In fact, properly immersed null holomorphic curves in $\mathbb{C}^{3}$ of arbitrary topology are constructed in Alarcón and López [1].

It is known that null curves in $\mathbb{C}^{3}$ are closely related to Legendrian curves in $\mathbb{C}^{3}$ (see Bryant [2] and also Ejiri-Takahashi [3] for the corresponding SL(2, $\mathbb{C}$ ) case). It can easily be checked that a holomorphic immersion $\mathcal{L}: \mathbb{D}_{1} \rightarrow \operatorname{SL}(2, \mathbb{C})$ is Legendrian if $\mathcal{L}^{-1} d \mathcal{L}$ is off-diagonal, namely, there exist two holomorphic 1 -forms $\omega$ and $\theta$ on $\mathbb{D}_{1}$ such that

$$
\mathcal{L}^{-1} d \mathcal{L}=\left(\begin{array}{cc}
0 & \theta \\
\omega & 0
\end{array}\right)
$$

As pointed out in the above paragraph, the projection of $\mathcal{L}$ into hyperbolic 3 -space gives a flat front in $H^{3}=\mathrm{SL}(2, \mathbb{C}) / \mathrm{SU}(2)$. Then the singular set of this flat surface in $H^{3}$ is given by

$$
\left\{z \in \mathbb{D}_{1} ;|\rho(z)|=1\right\}
$$

where $\rho$ is the meromorphic function defined by $\rho:=\theta / \omega$, called the ratio of canonical forms (cf. [10] and [11]). By Darboux's theorem, the contact structure of $\operatorname{SL}(2, \mathbb{C})$ is locally equivalent to that of $\mathbb{C}^{3}$. Moreover, the explicit transformation

$$
\mathcal{T}: \mathbb{C}^{3} \ni(X, Y, Z) \longmapsto\left(\begin{array}{cc}
e^{-Z} & Y e^{Z} \\
X e^{-Z} & (1+X Y) e^{Z}
\end{array}\right) \in \mathrm{SL}(2, \mathbb{C})
$$

maps holomorphic Legendrian curves in $\mathbb{C}^{3}$ with respect to the contact form

$$
\Omega_{C}:=d Z+Y d X
$$

to holomorphic Legendrian curves in $\mathrm{SL}(2, \mathbb{C})$. Using this transformation, we prove the following assertion. 
Theorem. There exists a weakly complete flat front in hyperbolic 3-space whose induced pair of hyperbolic Gauss maps have images contained in an arbitrarily given open disk in the ideal boundary of $H^{3}$.

It should be remarked that there are no compact flat fronts in $H^{3}$ (see Proposition 3.6 in [10]). Also, the assumption of weak completeness in the theorem is crucial, since the two hyperbolic Gauss maps can omit at most finitely many points if the given flat front is complete (see Remark 2.5). In contrast to this theorem, Kawakami [8] showed that the ratio of canonical forms $\rho$ of weakly complete flat fronts can omit at most three exceptional values.

\section{Proof of the theorem}

This section is devoted to proving the theorem stated in the introduction.

Recall that

$$
H^{3}:=\mathrm{SL}(2, \mathbb{C}) / \mathrm{SU}(2)=\left\{a a^{*} ; a \in \mathrm{SL}(2, \mathbb{C})\right\} \quad\left(a^{*}={ }^{t} \bar{a}\right) .
$$

is the hyperbolic 3 -space of constant curvature -1. A smooth map $f: \mathbb{D}_{1} \rightarrow H^{3}$ is called a (wave) front if there exists a Legendrian immersion $L_{f}: \mathbb{D}_{1} \rightarrow T_{1}^{*} H^{3}$ with respect to the canonical contact structure of the unit cotangent bundle $\pi: T_{1}^{*} H^{3} \rightarrow$ $H^{3}$ such that $\pi \circ L_{f}=f$. For a holomorphic Legendrian immersion $\mathcal{L}: \mathbb{D}_{1} \rightarrow \mathrm{SL}(2, \mathbb{C})$, the projection

$$
f:=\mathcal{L} \mathcal{L}^{*}: \mathbb{D}_{1} \longrightarrow H^{3}
$$

gives a flat front in $H^{3}$ (see [10] and [11] for the definition of flat fronts). In particular, the Gaussian curvature vanishes at each point where $f$ is an immersion. We call $\mathcal{L}$ in $(2.2)$ the holomorphic lift of $f$. A flat front $f$ is called weakly complete if its holomorphic lift is complete with respect to the pull-back metric

$$
d s_{\mathcal{L}}^{2}=|\omega|^{2}+|\theta|^{2} \quad\left(|\omega|^{2}:=\omega \bar{\omega},|\theta|^{2}:=\theta \bar{\theta}\right)
$$

of the canonical Hermitian metric of $\operatorname{SL}(2, \mathbb{C})$ by $\mathcal{L}$, where $\omega$ and $\theta$ are holomorphic 1-forms satisfying (1.3) and defined on $\mathbb{D}_{1}$ (cf. [10] and [11]). The first fundamental form of $f$ is written as

$$
d s_{f}^{2}:=|\omega|^{2}+|\theta|^{2}+\omega \theta+\bar{\omega} \bar{\theta}=|\omega+\bar{\theta}|^{2} .
$$

On the other hand, the pair of hyperbolic Gauss maps of $f$ is given by

$$
G_{+}:=\frac{A}{C}, \quad G_{-}:=\frac{B}{D},
$$

where

$$
\mathcal{L}:=\left(\begin{array}{cc}
A & B \\
C & D
\end{array}\right)
$$

We now prove the theorem in the introduction. Let

$$
F=(X, Y, Z): \mathbb{D}_{1} \longrightarrow \mathbb{C}^{3}
$$

be a bounded null ${ }^{1}$ holomorphic immersion whose induced metric is complete.

\footnotetext{
${ }^{1}$ We say that the immersion $F$ is null if it satisfies $d X^{2}+d Y^{2}+d Z^{2}=0$.
} 
Without loss of generality, we may assume that

$$
1<|X|<2, \quad|Y|<\frac{1}{3}
$$

hold on $\mathbb{D}_{1}$. Let $(g, \eta d z)$ be the Weierstrass data of $F$, that is,

$$
F_{z}\left(:=\frac{d F}{d z}\right)=\frac{1}{2}\left(1-g^{2}, \sqrt{-1}\left(1+g^{2}\right), 2 g\right) \eta .
$$

The metric induced by $F$ is written as

$$
|d X|^{2}+|d Y|^{2}+|d Z|^{2}=\frac{1}{2}\left(1+|g|^{2}\right)^{2}|\eta d z|^{2} .
$$

By (2.6), the projection $\hat{F}:=(X, Y): \mathbb{D}_{1} \rightarrow \mathbb{C}^{2}$ of $F$ is a bounded holomorphic map. Moreover, the following assertion holds:

Lemma 2.1. The metric

$$
d \sigma^{2}:=|d X|^{2}+|d Y|^{2},
$$

induced by $\hat{F}$, is a complete Riemannian metric on $\mathbb{D}_{1}$. In particular, $\hat{F}$ is a holomorphic immersion.

Proof. We have that

$$
\begin{aligned}
2 d \sigma^{2} & =2\left|\hat{F}_{z}\right|^{2}|d z|^{2}=\frac{1}{2}\left(\left|1-g^{2}\right|^{2}+\left|1+g^{2}\right|^{2}\right)|\eta d z|^{2}=\left(1+|g|^{4}\right)|\eta d z|^{2} \\
& \geq \frac{1}{2}\left(1+|g|^{2}\right)^{2}|\eta d z|^{2}=\left|F_{z}\right|^{2}|d z|^{2}=|d X|^{2}+|d Y|^{2}+|d Z|^{2} .
\end{aligned}
$$

Since $F$ is a complete immersion, $d \sigma^{2}$ is a complete Riemannian metric.

We now consider a new holomorphic immersion defined by

$$
\tilde{F}:=(X, Y, W): \mathbb{D}_{1} \longrightarrow \mathbb{C}^{3}, \quad \text { where } W:=-\int_{0}^{z} Y d X .
$$

Then $\tilde{F}$ gives a holomorphic Legendrian immersion with respect to the contact form $\Omega_{C}$ as in (1.5). Then the induced map (see (1.4) for the definition of $\mathcal{T}$ )

$$
\mathcal{L}:=\mathcal{T} \circ \tilde{F}: \mathbb{D}_{1} \longrightarrow \mathrm{SL}(2, \mathbb{C})
$$

can be written as

$$
\mathcal{L}=\left(\begin{array}{cc}
e^{-W} & Y e^{W} \\
X e^{-W} & (1+X Y) e^{W}
\end{array}\right),
$$

and the mapping $f: \mathbb{D}_{1} \rightarrow H^{3}$ given by (2.2) is a flat front. In fact, by a straightforward calculation, we have that

$$
\mathcal{L}^{-1} d \mathcal{L}=\left(\begin{array}{cc}
-(Y d X+d W) & e^{2 W}\left(d Y-Y^{2} d X\right) \\
e^{-2 W} d X & Y d X+d W
\end{array}\right)
$$

Since $Y d X+d W=\tilde{F}^{*} \Omega_{C}$ vanishes identically, the $\operatorname{sl}(2, \mathbb{C})$-valued 1 -form $\mathcal{L}^{-1} d \mathcal{L}$ is off-diagonal (i.e., we just checked that $\mathcal{L}$ is Legendrian), where $\operatorname{sl}(2, \mathbb{C})$ denotes the set of traceless $2 \times 2$ matrices. The following assertion holds: 
Proposition 2.2. The images of the two hyperbolic Gauss maps $G_{+}$and $G_{-}$ associated to $f$ lie in the unit disk $\{\xi \in \mathbb{C}:|\xi|<1\}$.

Proof. By (2.5) and (2.8), we have that $G_{+}=1 / X$ and $G_{-}=Y /(1+X Y)$. The inequalities (2.6) yield the assertion.

To prove the completeness of the metric $d s_{\mathcal{L}}^{2}$, we need the following assertion:

Lemma 2.3. The metric $d s_{\mathcal{L}}^{2}$ is positive definite and satisfies the inequality

$$
d s_{f}^{2} \leq 2 d s_{\mathcal{L}}^{2} .
$$

Proof. Since $\mathcal{T}$ is a local diffeomorphism, $\mathcal{L}$ is an immersion and $d s_{\mathcal{L}}^{2}$ is positive definite. The inequality (2.10) is obtained as follows (see (2.3) and (2.4)):

$$
d s_{f}^{2}=|\omega|^{2}+|\theta|^{2}+\omega \theta+\bar{\omega} \bar{\theta} \leq 2|\omega||\theta|+|\omega|^{2}+|\theta|^{2} \leq 2\left(|\omega|^{2}+|\theta|^{2}\right)=2 d s_{\mathcal{L}}^{2} .
$$

The following assertion is a key to proving the theorem.

Proposition 2.4. The metric $d s_{\mathcal{L}}^{2}$ is complete.

Proof. We fix an arbitrary piecewise smooth divergent path $\gamma:[0, \infty) \rightarrow \mathbb{D}_{1}$. It is sufficient to show that the image of $\gamma$ has infinite length with respect to $d s_{\mathcal{L}}^{2}$. If $f(\gamma([0, \infty)))$ is unbounded in $H^{3}$, then the path $\gamma$ must have infinite length with respect to $d s_{f}^{2}$ because of the completeness of the hyperbolic space $H^{3}$. Then the inequality (2.10) implies that $\gamma$ has infinite length with respect to $d s_{\mathcal{L}}^{2}$. So we assume that $f(\gamma([0, \infty)))$ is bounded in $H^{3}$. Since $\mathrm{SU}(2)$ is compact, (2.1) yields that the image $\mathcal{L}(\gamma([0, \infty)))$ is bounded in $\mathrm{SL}(2, \mathbb{C})$. By $(2.8)$, there exists a positive constant $m$ such that

$$
\left|e^{-W}\right|<m \quad \text { and } \quad\left|(1+X Y) e^{W}\right|<\frac{m}{3}
$$

hold on $\gamma$. Using (2.6), we have that

$$
\frac{1}{m}<\left|e^{W(\gamma(t))}\right|<m \quad(t \geq 0) .
$$

On the other hand, there holds that (see (2.9))

$$
\mathcal{L}^{-1} d \mathcal{L}=\left(\begin{array}{cc}
0 & e^{2 W}\left(d Y-Y^{2} d X\right) \\
e^{-2 W} d X & 0
\end{array}\right) .
$$

By (2.3), along $\gamma$ there holds

$$
\begin{aligned}
d s_{\mathcal{L}}^{2} & =\left|e^{-4 W}\right||d X|^{2}+\left|e^{4 W}\right|\left|d Y-Y^{2} d X\right|^{2}>\frac{1}{m^{4}}\left(|d X|^{2}+\left|d Y-Y^{2} d X\right|^{2}\right) \\
& \geq \frac{1}{m^{4}}\left(|d X|^{2}+|d Y|^{2}-2\left(2\left|Y^{2} d X\right|\right) \frac{|d Y|}{2}+|Y|^{4}|d X|^{2}\right) \\
& \geq \frac{1}{m^{4}}\left(|d X|^{2}+|d Y|^{2}-\left(4\left|Y^{2} d X\right|^{2}+\frac{|d Y|^{2}}{4}\right)+|Y|^{4}|d X|^{2}\right) \\
& =\frac{1}{m^{4}}\left(\left(1-3|Y|^{4}\right)|d X|^{2}+\frac{3|d Y|^{2}}{4}\right) .
\end{aligned}
$$


Using (2.6) again,

$$
d s_{\mathcal{L}}^{2}>\frac{1}{m^{4}}\left(\frac{26|d X|^{2}}{27}+\frac{3|d Y|^{2}}{4}\right)>\frac{3}{4 m^{4}}\left(|d X|^{2}+|d Y|^{2}\right)=\frac{3}{4 m^{4}} d \sigma^{2}
$$

holds on $\gamma$. Since $d \sigma^{2}$ is a complete metric (see Lemma 2.1), we get the conclusion.

Proof of the theorem. Let $r$ be a sufficiently small positive number. We set

$$
\mathcal{L}_{r}:=\left(\begin{array}{cc}
r & 0 \\
0 & r^{-1}
\end{array}\right) \mathcal{L} .
$$

Then $f_{r}:=\mathcal{L}_{r} \mathcal{L}_{r}^{*}$ is the desired flat front. In fact, $f_{r}$ is weakly complete, since the induced metric of $\mathcal{L}_{r}$ is complete (see Proposition 2.4). On the other hand, the hyperbolic Gauss maps of $f_{r}$ are equal to $r^{2} G_{+}$and $r^{2} G_{-}$, and their images are contained in a disk of radius $r^{2}$ (see Proposition 2.2).

Remark 2.5. Let $\left(G_{+}, G_{-}\right)$be the pair of hyperbolic Gauss maps induced by a complete flat front $f: M^{2} \rightarrow H^{3}$, where $M^{2}$ is a Riemann surface. (For the definition of completeness, see page 150 of [10].) By Lemma 3.3 of [10], there exists a closed Riemann surface $\bar{M}^{2}$ and a finite number of points $p_{1}, \ldots, p_{n}$ in $\bar{M}^{2}$ such that $M^{2}$ is biholomorphic to $\bar{M}^{2} \backslash\left\{p_{1}, \ldots, p_{n}\right\}$. If $G_{+}$(resp. $G_{-}$) has an essential singularity at some $p_{j}$ (i.e. $p_{j}$ is an irregular end), Picard's theorem implies that $G_{+}$(resp. $G_{-}$) omits at most two points in $\mathbb{C} \cup\{\infty\}$. Otherwise, both $G_{+}$and $G_{-}$are meromorphic functions on a compact Riemann surface $\bar{M}^{2}$. Hence both $G_{+}$and $G_{-}$are surjective maps onto $\mathbb{C} \cup\{\infty\}$.

We now remark that the following problem seems interesting as an analogue of the Calabi-Yau problem in minimal surface theory:

Question. Is there a bounded holomorphic Legendrian curve immersed in $\mathbb{C}^{3}$ which is complete with respect to the canonical Hermitian metric of $\mathbb{C}^{3}$ ?

If the question had an affirmative answer, then one could prove the existence of weakly complete bounded flat fronts in $H^{3}$ as follows. Using (1.4), the existence of such Legendrian curves in $\mathbb{C}^{3}$ implies the existence of bounded holomorphic Legendrian curves in $\mathrm{SL}(2, \mathbb{C})$. Hence, by applying Lemma 3.1 in [13], one can easily check that such holomorphic curves in $\mathrm{SL}(2, \mathbb{C})$ are complete with respect to the bi-invariant Hermitian metric of $\mathrm{SL}(2, \mathbb{C})$. In particular, their projections into $H^{3}=\mathrm{SL}(2, \mathbb{C}) / \mathrm{SU}(2)$ gives weakly complete bounded flat surfaces.

On the other hand, the above question is also related to our main result. Moreover, it is also closely related to the existence of bounded weakly complete improper affine fronts in the affine space $\mathbb{R}^{3}$ : a notion of $I A$-maps in the affine 3 -space has been introduced by A. Martínez [13]. IA-maps are improper affine spheres with a certain kind of singularity. Since all IA-maps are wave fronts (see [15], [16]), we call them improper affine fronts (the terminology 'improper affine fronts' has already been used by Kawakami-Nakajo, [9]). The precise definition of improper affine fronts is given in Remark 4.3 of [16]. In [16], weak completeness of improper affine fronts is introduced. Then we have: 
Proposition 2.6. The existence of a complete bounded immersed Legendrian curve as in the above question would imply the existence of a weakly complete improper affine front whose image is bounded.

Proof. Let $F=(X, Y, Z)$ be a complete bounded Legendrian immersion into $\mathbb{C}^{3}$. Since $F$ is Legendrian, (1.5) yields that $d Z=-Y d X$. Here, by completeness of $F$, the induced metric

$d s_{F}^{2}=|d X|^{2}+|d Y|^{2}+|d Z|^{2}=|d X|^{2}+|d Y|^{2}+|Y d X|^{2}=\left(|Y|^{2}+1\right)|d X|^{2}+|d Y|^{2}$

is complete. Moreover, since the image of $F$ is bounded, we have

$$
d s_{F}^{2} \leq C\left(|d X|^{2}+|d Y|^{2}\right) \quad(C>0 \text { is a constant }) .
$$

Thus, the metric

$$
d \tau^{2}:=|d X|^{2}+|d Y|^{2}
$$

is complete. Hence, we have the following improper affine front $f$, substituting the pair of holomorphic functions $(X, Y)$ into Martínez's representation formula (see Theorem 3 in [13]),

$$
\begin{aligned}
f & =\left(X+\bar{Y}, \frac{1}{2}\left(|X|^{2}-|Y|^{2}\right)+\operatorname{Re}\left(X Y-2 \int Y d X\right)\right) \\
& =\left(X+\bar{Y}, \frac{1}{2}\left(|X|^{2}-|Y|^{2}\right)+\operatorname{Re}(X Y+2 Z)\right): \mathbb{D}_{1} \rightarrow \mathbb{R}^{3} .
\end{aligned}
$$

Since $d \tau^{2}$ in $(2.12)$ is complete, $f$ is weakly complete, by the definition of weak completeness given in [16]. The boundedness of $f$ follows from that of $F$.

\section{References}

[1] Alarcón, A. And López, F. J.: Null curves in $\mathbb{C}^{3}$ and Calabi-Yau conjectures. Math. Ann. 355 (2013), no. 2, 429-455.

[2] Bryant, R. L.: Surfaces in conformal geometry. In The mathematical heritage of Hermann Weyl (Durham, NC, 1987), 227-240. Proceedings of Symposia in Pure Mathematics 48, Amer. Math. Soc., Providence, RI, 1988.

[3] EjiRi, N. and Takahashi, M.: The Lie transform between null curves in $\operatorname{SL}(2, \mathbb{C})$ and contact curves in $\operatorname{PSL}(2, \mathbb{C})$. In Riemann surfaces, harmonic maps and visualization, 265-277. OCAMI Studies 3, Osaka Munic. Univ. Press, Osaka, 2010.

[4] Ferrer, L., Martín, F., Umehara, M. and Yamada, K.: A construction of a complete bounded null curve in $\mathbb{C}^{3}$. To appear in Kodai Math. J.

[5] Fujimoto, H.: Value distribution of the Gauss map of complete minimal surfaces in $\mathbb{R}^{m}$. J. Math. Soc. Japan 35 (1983), 663-681.

[6] Fujimoto, H.: On the number of exceptional values of the Gauss maps of minimal surfaces. J. Math. Soc. Japan 40 (1988), 235-247. 
[7] Gálvez, J. A., Martínez, A. And Milán, F.: Flat surfaces in the hyperbolic 3-space. Math. Ann. 316 (2000), 419-435.

[8] KaWAKami, Y.: A ramification theorem for the ratio of canonical forms of flat surfaces in hyperbolic 3-space. To appear in Geom. Dedicata. DOI: 10.1007/s10711-013-9904-8.

[9] Kawakami, Y. and Nakajo, D.: Value distribution of the Gauss map of improper affine spheres. J. Math. Soc. Japan 64 (2012), 799-821.

[10] Kokubu, M., Umehara, M. and Yamada, K.: Flat fronts in hyperbolic 3-space. Pacific J. Math. 216 (2004), 149-175.

[11] Kokubu, M., Rossman, W., Umehara, M. and Yamada, K.: Flat fronts in hyperbolic 3-space and their caustics., J. Math. Soc. Japan 59 (2007), 265-299.

[12] Kokubu, M., Rossman, W., Umehara, M. and Yamada, K.: Asymptotic behavior of flat surfaces in hyperbolic 3-space. J. Math. Soc. Japan 61 (2009), 799-852.

[13] Martínez, A.: Improper affine maps. Math. Z. 249 (2005), 755-766.

[14] Nadirashvili, N.: Hadamard's and Calabi-Yau's conjectures on negatively curved and minimal surfaces. Invent. Math. 126 (1996), 457-465.

[15] NAKAJO, D.: A representation formula for indefinite improper affine spheres. Results Math. 55 (2009), 139-159.

[16] Umehara, M. and Yamada, K.: Applications of a completeness lemma in minimal surface theory to various classes of surfaces. Bull. London Math. Soc. 43 (2011), 191-199.

[17] Yu, Z.-H.: The value distribution of the hyperbolic Gauss map. Proc. Amer. Math. Soc. 125 (1997), 2997-3001.

Received May 12, 2012.

Francisco Martín: Departamento de Geometría y Topología, Universidad de Granada, 18071 Granada, Spain.

E-mail: fmartin@ugr.es

Masanki Umehara: Department of Mathematical and Computing Sciences, Tokyo Institute of Technology 2-12-1-W8-34, O-okayama, Meguro-ku, Tokyo 152-8552, Japan.

E-mail: umehara@is.titech.ac.jp

Kotaro Yamada: Department of Mathematics, Tokyo Institute of Technology 2-121-H-7, O-okayama, Meguro-ku, Tokyo 152-8551, Japan.

E-mail: kotaro@math.titech.ac.jp

The first author is partially supported by MEC-FEDER Grants no. MTM2007-61775 and no. MTM2011-22547, and a Regional J. Andalucía Grant no. P09-FQM-5088. The second and the third authors are partially supported by a Grant-in-Aid for Scientific Research (A) no. 22244006, and Scientific Research (B) no. 21340016, respectively, from the Japan Society for the Promotion of Science. 